\title{
A Chandra survey of fluorescence Fe lines in X-ray binaries at high resolution
}

\author{
J.M. Torrejón $* a \dagger$ N.S. Schulz ${ }^{b}$, M.A. Nowak ${ }^{b}$ and T.R. Kallman ${ }^{c}$ \\ ${ }^{a}$ Instituto de Física Aplicada a las Ciencias y las Tecnologías, Universidad de Alicante, Spain \\ ${ }^{b}$ MIT Kavli Institute for Astrophysics and Space Research, Cambridge MA, USA \\ ${ }^{c}$ NASA Goddard Space Flight Center, Greenbelt MD, USA \\ E-mail: jmt@ua.es, nss@space.mit.edu, mnowak@space.mit.edu, \\ tymothy.r.kallmanegsfc.nasa.gov
}

In this paper we present a comprehensive survey of $41 \mathrm{X}$-ray binaries (10 HMXBs and 31 LMXBs) with Chandra with specific emphasis on the Fe $\mathrm{K}$ region and the narrow $\mathrm{Fe} \mathrm{K} \alpha$ line, at the highest resolution possible. We find that: $a$ ) The $\mathrm{Fe} \mathrm{K} \alpha$ line is always centered at $\lambda=1.9387 \pm 0.0016 \AA$, compatible with Fe I up to Fe VIII; we detect no shifts to higher ionization states nor any difference between HMXBs and LMXBs. $b$ ) The line is very narrow, with $F W H M \leq 5 \mathrm{~m} \AA$, normally not resolved by Chandra which means that the reprocessing material is not rotating at high speeds. 1c) Fe K $\alpha$ fluorescence is present in all the HMXB in the survey. In contrast, such emissions are astonishingly rare $(\sim 10 \%)$ among low mass $\mathrm{X}$-ray binaries (LMXB). d) The lack of Fe line emission is always accompanied by the lack of any detectable $\mathrm{K}$ edge. $e$ ) We obtain the empirical curve of growth of the equivalent width of the $\mathrm{Fe} \mathrm{K} \alpha$ line versus the density column of the reprocessing material, i.e. $E W_{\mathrm{K} \alpha}$ vs $N_{\mathrm{H}}$, and show that it is consistent with a reprocessing region spherically distributed around the compact object. $f$ ) We show that fluorescence in X-ray binaries follows the X-ray Baldwin effect as previously only found in the $\mathrm{X}$-ray spectra of active galactic nuclei. We interpret this finding as evidence of decreasing neutral $\mathrm{Fe}$ abundance with increasing X-ray illumination and use it to explain some spectral states of Cyg $\mathrm{X}-1$ and as a possible cause of the lack of narrow Fe line emission in LMXBs.

8th INTEGRAL Workshop "The Restless Gamma-ray Universe"- Integral2010,

September 27-30, 2010

Dublin Ireland

\footnotetext{
* Speaker.

${ }^{\dagger}$ Partially suported by AYA2008-06166-C03-03 and AYA2010-15431
} 


\section{Introduction}

Fluorescence excitation occurs whenever there is a low ionization gas illuminated by X-rays. In X-ray binaries (XRBs), the strong point like source of X-rays, powered via accretion, allows to observe strong fluorescence emission from the irradiated circumstellar material, either the wind or the accretion disk. Whenever this material is more neutral than Li-like, the Fe atoms present in the stellar wind absorb a significant fraction of continuum photons blueward of the K edge (at $\sim 1.74$ $\AA$ ) thereby removing $\mathrm{K}$ shell electrons. The vacancy thus produced will be occupied by electrons from the upper levels producing $\mathrm{K} \alpha(\mathrm{L} \rightarrow \mathrm{K})$ and $\mathrm{K} \beta(\mathrm{M} \rightarrow \mathrm{K})$ fluorescence emission lines at $~$ $1.94 \AA$ and $1.75 \AA$ respectively. Since the Fe is abundant and it appears in an unconfused part of the spectrum, Fe $\mathrm{K}$ fluorescence is observed in a wide range of objects and has been used as a primary tool to study the material in the vicinity of X-ray sources.

[4] established a comprehensive catalog of Fe line sources using EXOSAT GSPC. These authors were able to detect iron line emission in 51 sources out of which 32 were identified as X-ray binaries (XRB). From these, $20(\sim 63 \%)$ were LMXB and $12(\sim 37 \%)$ HMXB. On average, the former showed a broad $(\sim 1 \mathrm{keV})$ line centered at $6.628 \pm 0.012 \mathrm{keV}$, while the latter tended to show narrower $(\sim 0.5 \mathrm{keV})$ lines centered at $6.533 \pm 0.003 \mathrm{keV}$. EXOSAT GSPC had a spectral resolution of about this amount and, therefore, a width of $\sim 0.5 \mathrm{keV}$ represents an upper limit. Later [1] performed a study of the Fe K line in a sample of 20 LMXB using ASCA GIS and SIS data. These authors were able to detect significant Fe line emission in roughly half of the sources. This line tended to be centered around $6.6 \mathrm{keV}$ but showed large scatter with extreme values going from $\sim 6.55$ to $6.7 \mathrm{keV}$. In general, the FWHM is not resolved but for those sources where the width could be measured was $\sim 0.5 \mathrm{keV}$. Recently, [5] have performed a comprehensive survey of broad Fe lines in 16 LMXBs using XMM-Newton PN. These authors find the line in $80 \%$ fo the sources with average parameters $E=6.67 \pm 0.02 \mathrm{keV}$ and $F W H M \sim 0.78 \pm 0.05 \mathrm{keV}$.

In the present survey [8] we have reprocessed and analyzed data from 41 XRBs (= $10 \mathrm{HMXBs}$ + 31 LMXBs) at the highest resolution possible using Chandra gratings with specific emphasis in the narrow component.

\section{The Observations}

In Fig. 1 we present four representative examples of the quality of the fits. The upper row shows two HMXBs of our sample, Vela X-1 (left) and Cygnus X-3 (right). As can be seen, the spectra of Vela X-1 is relatively clean, showing only $\mathrm{Fe} \mathrm{K} \alpha, \mathrm{Fe} \mathrm{K} \beta$ and the $\mathrm{K}$ edge (plus a small hot line beyond the edge). The spectra of Cygnus X-3, in turn, is more complex. It shows two hot lines, Fe XXV and Fe XXVI of ionized iron. These latter lines, in particular Fe XXV, can not be resolved in low resolution and broad band spectra and are often confused with the true fluorescence Fe $\mathrm{K} \alpha$ of (near)neutral iron. Hence the paramount importance of using Chandra for the present survey. The lower row corresponds to two LMXBs within our sample. The case of 4U1822-37 (left) shows a morphology similar to that of the HMXBs. Finally, we show the example of 4U1957+11 (right) which, as the vast majority of LMXBs, does not show any emission line or edge at all.

From the inspection of the fits these main conclusions can be drawn: 1) the narrow Fe line is present $\sim 100 \%$ of the HMXBs (10 out of 10$)$. In contrast it seems to be very rare $(\sim 13 \%)$ among 

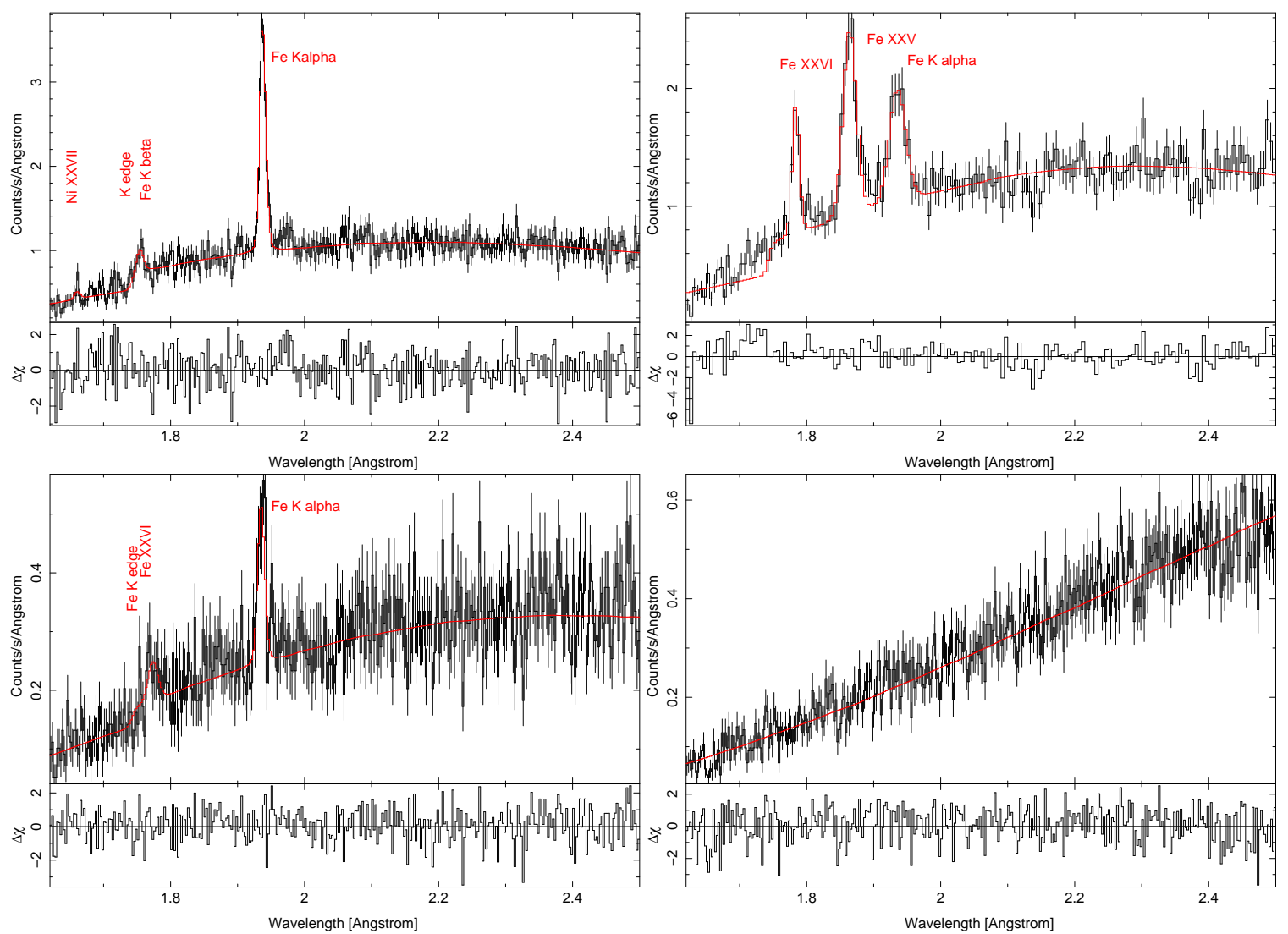

Figure 1: Four representative examples of the fitting process. Upper row: HMXBs. Left: Vela X-1 shows $\mathrm{Fe} \mathrm{K} \alpha$ and Fe K $\beta$ but not hot photoionization lines (except Ni XXVII). Right: Cyg X-3, in turn, shows hot photoionization lines of Fe XXV and Fe XXVI which, in low resolution and broadband spectra, are confused with the true cold $\mathrm{Fe} \mathrm{K} \alpha$ fluorescence line. Lower row: LMXBs. Left: 4U1822-37 shows Fe K $\alpha$ and a hot line. Right: The spectra of $4 \mathrm{U} 1957+11$, as the vast majority of LMXBs, shows no lines at all, in this case superimposed on a pure powerlaw continuum.

LMXBs (4 out of 31). On the other hand, the emission in HMXBs is very variable. The most striking case is that of Cyg X-1 where only two out of ten available observations showed emission. 2) The FeK $\alpha$ line is centered at $\lambda=1.9387 \pm 0.0016 \AA(E=6.391 \pm 0.005 \mathrm{keV})$ compatible with fluorescence from Fe I up to Fe VIII. We detect no shifts to higher ionization states nor any difference between HMXBs and LMXBs. The reprecessing material is (close to) neutral. 3) The $F W H M \leq 0.005 \AA$, very narrow, normally not resolved by Chandra. Therefore, the reprocessing material is not rotating at high speeds.

\section{Curve of Growth}

One of the goals of the this study is to establish an empirical relationship between $E W$ of the $\mathrm{Fe} \mathrm{K} \alpha$ fluorescence line and the column density of the reprocessing material and determine its Curve of Growth. In Fig 2 we plot the curve of growth of the $E W(K \alpha)$ with the H equivalent absorption column of the reprocessing material. As can be seen, the EWs of the Fe K $\alpha$ lines scale linearly with $N_{\mathrm{H}}$. The least squares best fit gives 

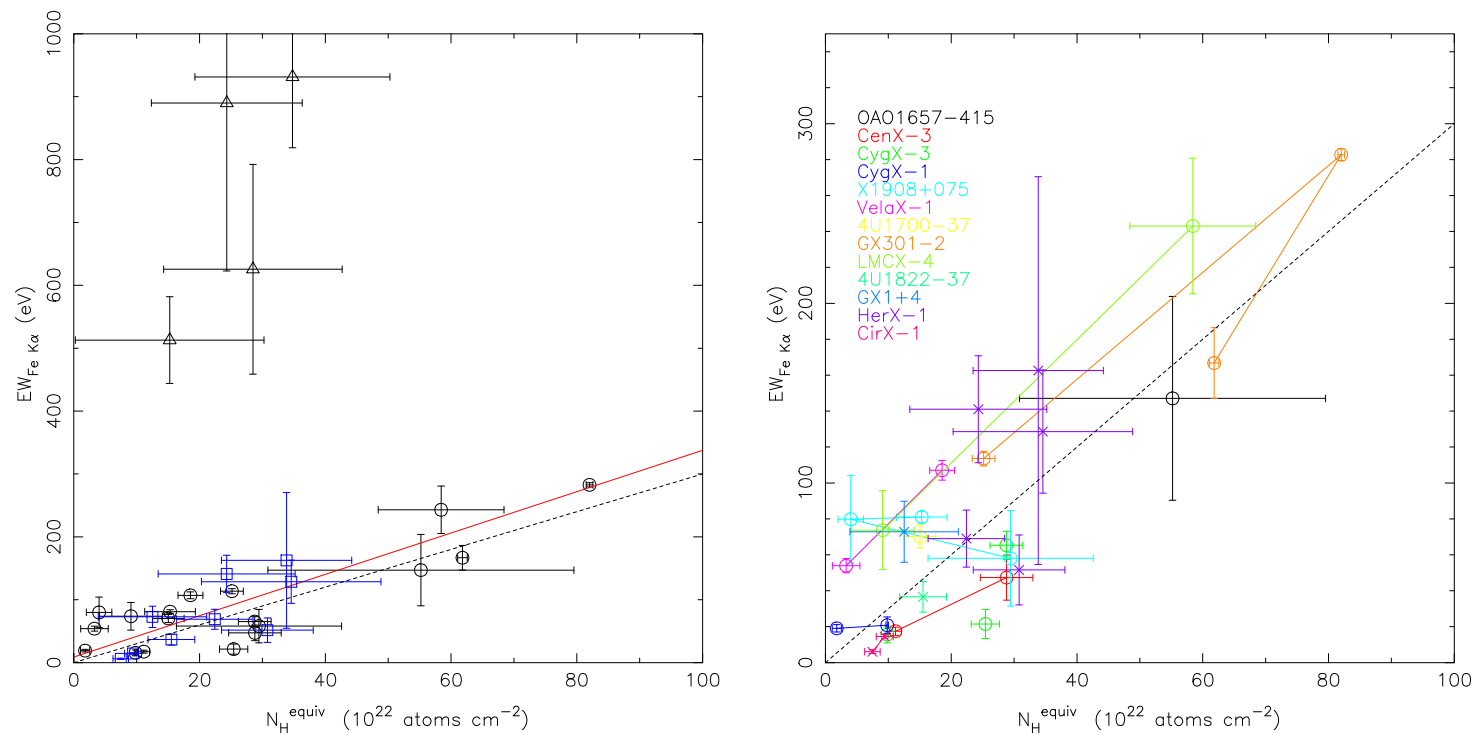

Figure 2: Curve of growth. The $E W$ of the Fe line grows with the column density of the reprocessing material (continuous red line) as predicted by the theoretical model of [6] for spherical geometry (dashed line). Black circles represent HMXB data and blue squares represent LMXB. Eclipse data (triangles, upper left corner) do not follow the main trend.

$$
E W_{F e K \alpha}(\mathrm{eV})=(3.29 \pm 0.05) N_{\mathrm{H}}^{22}
$$

where $N_{\mathrm{H}}^{22}$ is the equivalent $\mathrm{H}$ column of the reprocessing material in units of $10^{22}$ atoms $\mathrm{cm}^{-2}$. This relationship is plotted as a continuous red line in Fig 2. The degree of correlation is very high (Pearson correlation coefficient of $r=0.95$ ). We have also plotted the theoretical prediction given by [6], Eq.5, namely $E W(F e K \alpha) \simeq 3 N_{\mathrm{H}}^{22}[\mathrm{eV}]$ (black dashed line). The agreement is excellent even though there is a large scatter in the data. The available data, therefore, are consistent with a spherical distribution of the Fe fluorescence emission zone around the X-ray source. This implies a scenario where the compact object is deeply embedded within the stellar wind of the companion star, as is the likely case in most HMXBs. Likewise, no saturation effects are observed within the range of columns studied. There are points that deviate significantly from this trend (shown as triangles). These points correspond to eclipse data of Vela X-1 and LMC X-4. This can be explained taking into account that the the intensity of the line is normalized to the local continuum, which is strongly suppressed during eclipse. The line emitting gas is exposed to the full continuum from the compact object and we see the line emitting gas directly. However, we observe the continuum only via scattering. As a consequence, in eclipse, the very large $E W$ does not correlate well with $N_{\mathrm{H}}$.

\section{X-ray Baldwin effect}

While we observe a correlation of line flux with continuum flux, we also observe an anticorrelation of $E W$ with the same continuum flux (Fig. 3). Such an anticorrelation has been shown to 

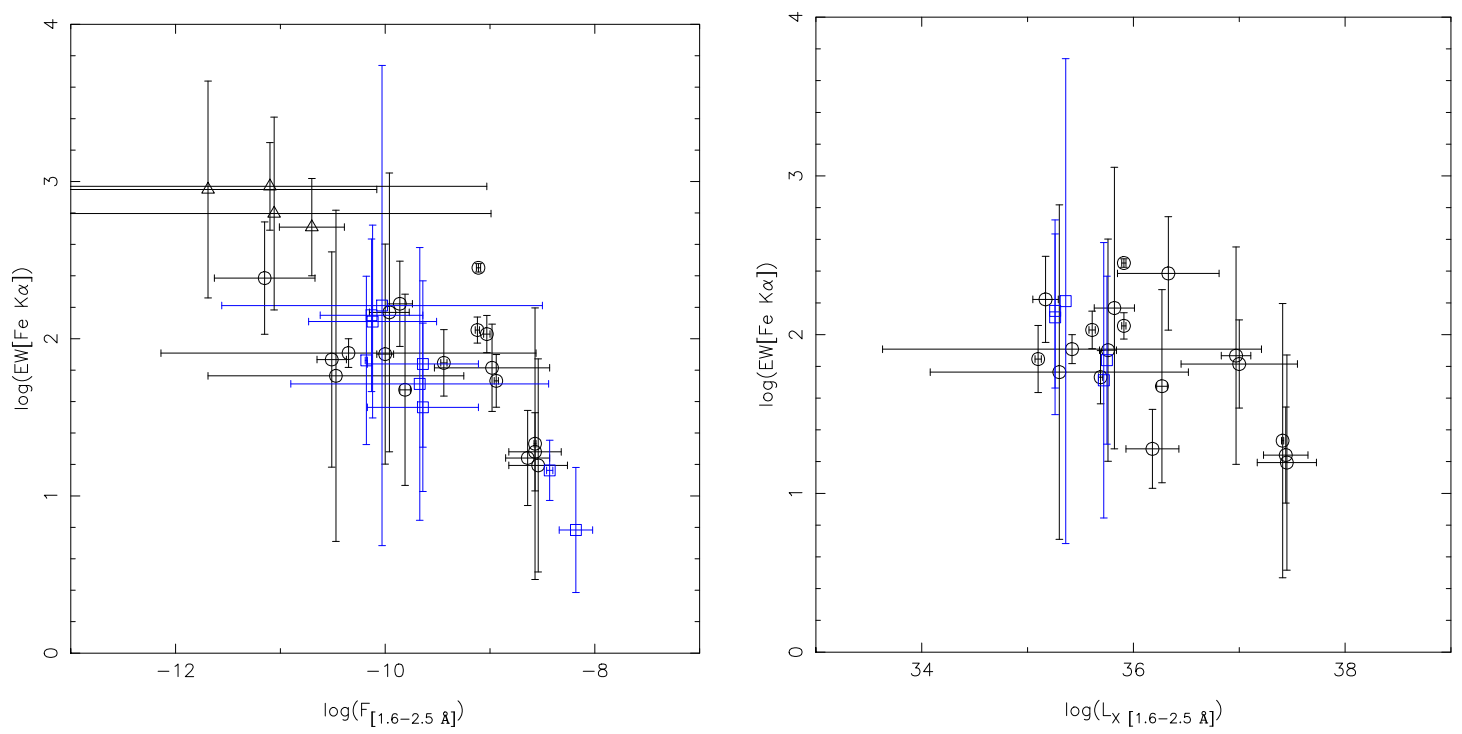

Figure 3: Left: Log-log plot of the EW of the Fe line vs the unabsorbed flux of the continuum. A clear anticorrelation can be seen. Black circles represent HMXB, blue squares LMXB and triangles the eclipse data $\left(E W_{\text {line }} \propto F_{\text {cont }}^{-0.437} ; r^{2}=0.80\right)$. Right: Baldwin effect for HMXB. Only sources with known distances have been included. An anticorrelation can be seen. Eclipse data have not been plotted $\left(E W_{\text {line }} \propto L_{X}^{-0.289} ; r^{2}=0.62\right)$

exist for AGNs exhibiting Fe K $\alpha$ fluorescence line. This phenomenon is called the 'X-ray Baldwin effect' following the discovery of a decrease in the EW of the CIV line with increasing UV luminosity in AGNs by [2]. Next we compute the intrinsic $L_{X}$ with the corresponding distance taken from the literature. Even though the trend is still visible, the correlation begins to break down. This can be due to a number of reasons: the distances for some systems are poorly known, the X-ray Baldwin is effective in the soft state but not in the hard state. Such issues can also contribute to the scatter in Fig. 3. These issues not withstanding, the Chandra data show, for the first time, that such a correlation seems to exist for X-ray binaries in general.

An immediate interpretation is that the reprocessing material becomes progressively ionized with increasing X-ray illumination, with the concurrent decrease in the Fe $\mathrm{K}$ shell fluorescence. This effect can explain the lack of Fe K $\alpha$ line in the high (soft) state of Cyg X-1. In LMXBs the lack of Fe $\mathrm{K} \alpha$ could mean disks too hot.

\section{Conclusions}

Regarding the narrow lines:

- The narrow Fe line is present $\sim 100 \%$ of the HMXBs, showing high varibility. In contrast it seems to be very rare $(\sim 13 \%)$ among LMXBs.

- The Fe $\mathrm{K} \alpha$ line is centered at $\lambda=1.9387 \pm 0.0016 \AA(E=6.391 \pm 0.005 \mathrm{keV})$ both for 


\begin{tabular}{llccc}
\hline & Line width & Abundance $(\%)$ & Energy $(\mathrm{keV})$ & Line Nature \\
\hline \hline LMXBs & narrow & $\sim 13$ & $6.391 \pm 0.005$ & Fe K $\alpha$ \\
& broad & $\sim 80$ & $6.67 \pm 0.02$ & Fe XXV? \\
HMXBs & narrow & $\sim 100$ & $6.391 \pm 0.005$ & Fe K $\alpha$ \\
& broad $^{a}$ & $?(<37)$ & $6.533 \pm 0.003$ & $?$ \\
\hline
\end{tabular}

Table 1: Summary of the characteristics of the narrow and broad lines in HMXBs and LMXBs. The narrow line data comes from the present survey while the broad line data comes from $\mathrm{Ng}$ et al. 2010 for LMXBs and Gottwald et al. 1995 for HMXB. In the last column we give a (tentative) identification about the possible nature of the line.

${ }^{a}$ Although narrower that for LMXBs

HMXBs and LMXBs, compatible with fluorescence from Fe I up to Fe VIII. The reprocessing material is (close to) neutral.

- The $F W H M \leq 0.005 \AA$, very narrow, normally not resolved by Chandra. Therefore, the reprocessing material is not rotating at high speeds.

- The reprocessing material follows a Curve of Growth compatible with spherically distributed material around the X-ray source.

- We observe the 'X-ray Baldwin Effect' in X-ray binaries. An immediate interpretation is that the reprocessing material becomes progressively ionized with increasing X-ray illumination.

Finally, the centroid energy of the broad lines (Table 1) suggest that they are probably not of fluorescence nature. The broad lines in LMXBs are more consistent with highly ionized Fe. This line could be produced much closer to the X-ray source where the broadening mechanisms will be much more efficient.

\section{References}

[1] Asai K., Dotani T., Nagase F. Mitsuda K. 2000, ApJS 131, 571

[2] Baldwin J.A. 1977, ApJ 214, 679

[3] Dunn R.J.H., Fender R.P., Körding E.G., Cabanac C. and Belloni T. 2008, MNRAS 387, 545

[4] Gottwald M., Parmar A. N., Reynolds A.P., White N.E. Peacock A., Taylor B.G. 1995, A\&AS 109, 9

[5] Ng C., Díaz Trigo M., Cadolle Bel M., Migliari S. 2010, A\&A 522, 96

[6] Kallman, T. Palmieri, P., Bautista M.A., Mendoza C., Krolik J.H. 2004, ApJS, 155, 675

[7] Palmeri P., Mendoza C., Kallman T.R., Bautista M.A., Meléndez M. 2003, A\&A 410, 359

[8] Torrejón J.M., Schulz N.S., Nowak M., Kallman, T. 2010, ApJ 715, 947 